\title{
Design and Fabrication of an End-Launched Rectangular Waveguide Adapter Fed by a Coaxial Loop
}

\author{
Doo-Yeong Yang, Member, KIICE \\ Department of Telecommunication Engineering, Jeju National University, Jeju 690-756, Korea
}

\begin{abstract}
An end-launched adapter combining a coaxial loop within a rectangular waveguide has been analyzed and designed. The accurate electromagnetic modeling of input impedance for the adapter design has been developed using a modal analysis method. Also, design parameters to improve the end-launched adapter have been investigated. Numerical and experimental results of the proposed model have been compared with the results of previous works, and verified by an electromagnetic simulator of High Frequency Structure Simulator (HFSS). The input voltage standing wave ratios (VSWRs) are smaller than 2 over the wide frequency band from $7.5 \mathrm{GHz}$ to $10.5 \mathrm{GHz}$.
\end{abstract}

Index Terms: Coaxial loop, End-launched adapter, Input impedance, Modal analysis method, Rectangular waveguide

\section{INTRODUCTION}

An end-launched rectangular waveguide adapter is used as an excitation element to transmit a modulated radio frequency (RF) signal to microwave devices such as a cavity filter and a horn antenna. The end-launched adapter consists of a rectangular waveguide and a coaxial loop shorted on the broad-wall of the waveguide. This adapter converts a coaxial mode to a waveguide dominant mode. This is known as a coaxial-to-waveguide transition and has been heavily researched. The basic theoretical procedure for the design of a coaxial-to-waveguide transition has been reported in [1-5]. However, these works could not obtain exact solutions because they applied approximation techniques to derive an expression of the input impedance.

This paper develops electromagnetic modeling of the input impedance to design an end-launched rectangular waveguide adapter by using the modal analysis method. This method gives a more accurate expression that does not use any approximation scheme for the input impedance, which is considered the dominant mode along with the higher-order modes inside a waveguide. Also, the result of the input impedance expression is analyzed and compared with the results of the previous work in [2, 4]. The validity of the proposed analytical model of input impedance is verified by an electromagnetic tool within High Frequency Structure Simulator (HFSS; ANSYS Inc, Canonsburg, PA, USA) based on a numerical method.

\section{LOOP COUPLING GEOMETRY OF END- LAUNCHED ADAPTER}

Fig. 1 shows the geometry of the end-launched rectangular waveguide adapter, where an inner conductor of the coaxial line is collinearly interconnected with the coupling loop shorted on the waveguide bottom. This type of a rectangular waveguide adapter converts the coaxial transverse electromagnetic (TEM) mode into the waveguide propagating mode. The radius of the inner conductor and the

Received 15 February 2012, Revised 05 March 2012, Accepted 21 March 2012

*Corresponding Author E-mail: yeongyd@jejunu.ac.kr 
radius of the outer conductor of the coaxial line are $c_{i}$ and $c_{0}$, respectively, and the inside of the coaxial line is filled with a material with a dielectric constant of $\varepsilon_{r}$. For the coupling loop, the z-directional current element is placed on the $\mathrm{z}$ axis $\left(0<\mathrm{z}<\mathrm{L}_{1}\right)$, while the $\mathrm{y}$-directional current element is placed on the $y$-axis $\left(0<y<L_{2}\right)$. The grounded position of the loop that is shorted on the waveguide broad-wall bottom is located at the point $\left(\mathrm{x}=\mathrm{x}_{\mathrm{o}}, \mathrm{y}=0, \mathrm{z}=\mathrm{L}_{1}\right)$. The modes inside a waveguide, which are excited by a coupling loop, are formed by two current elements. The transverse electric (TE) modes are only excited by the y-directional current element, while the transverse magnetic (TM) modes are excited by the z-directional current element of the loop. Therefore, hybrid modes exist in the end-launched adapter. The coupling loop dimension should be appropriately selected in order to convert the TEM mode of the coaxial line to the dominant $\mathrm{TE}_{10}$ mode inside the rectangular waveguide. The loop geometry has an especially large effect on the signal transmission, bandwidth, and return loss. To find the input impedance expression of the adapter, an analytical procedure is outlined in the following four steps [6]: First, sinusoidal trial current densities, which are the current distribution in the z-direction and the current distribution in the y-direction, are defined. Second, the magnetic vector potentials due to the current densities are derived. Third, the expression of the electric field without any approximation scheme inside a rectangular waveguide is obtained from the magnetic vector potentials using the Lorentz gauge condition [7]. Finally, the exact solution of the input impedance for the coaxial-to-waveguide endlaunched adapter is derived by applying the energy reciprocity theorem from electromagnetic field theory [8].

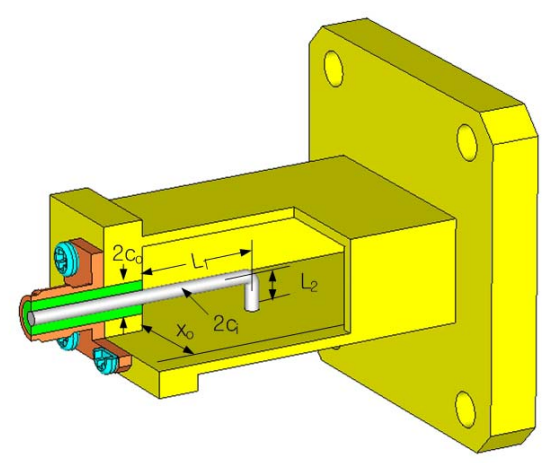

Fig. 1. Geometry of end-launched rectangular waveguide adapter.

The modal analysis expression for the input impedance can be obtained as

$$
\begin{aligned}
Z_{\text {in }}= & \frac{1}{4 \pi^{2} a b \cos ^{2}\left(k\left(L_{1}+L_{2}\right)\right)} \\
& {\left[\sum_{m=1}^{\infty} \sum_{n=0}^{\infty}\left\{\frac{\varepsilon_{n} k^{2}}{\gamma} A_{m n}^{2} \sin \left(\frac{m \pi x_{o}}{a}\right)\left(B_{m n, 1}+B_{m n, 2}\right) C_{m n} D_{m n}\right\}\right.} \\
& \left.-\sum_{m=1}^{\infty} \sum_{n=1}^{\infty}\left\{\frac{E_{m n}^{2}}{\gamma\left(k^{2}+\gamma^{2}\right)}\left(F_{m n, 1}+F_{m n, 2}+F_{m n, 3}\right)\right\}\right]
\end{aligned}
$$

where $k$ and $\gamma$ are the propagation constant in free space and inside the rectangular waveguide, respectively. $a b$ is a waveguide cross-section. $\varepsilon_{n}=1$ for $\mathrm{n}=0$ and $\varepsilon_{n}=2$ otherwise. $A_{m n}, B_{m n, 1}, B_{m n, 2}, C_{m n}, D_{m n}, E_{m n}, F_{m n, 1}, F_{m n, 2}$, and $\mathrm{F}_{\mathrm{mn}, 3}$ are mentioned in Appendix A-1 to A-9.

The real component of the input impedance of the endlaunched adapter is attributed to the dominant mode; the higher order modes only contribute to the reactive component because of evanescent modes. The shorted perpendicular arm correlates with the longitudinal arm of the loop as shown in Fig. 1, and from this, the real component of input impedance can be matched to the characteristic impedance of the coaxial line and the reactive component can be canceled out. The best performance of the end-launched adapter is achieved when the reactance cancellation occurs over the broadband operating frequency and the resistance is matched to the input impedance of the adapter connected by the coaxial line.

The input reflection coefficient and the input voltage standing wave ratio (VSWR) from (1) are defined as

$$
\begin{gathered}
|\Gamma|=\frac{\sqrt{\left(\operatorname{Re}\left(Z_{\text {in }}\right)-Z_{0}\right)^{2}+\left(\operatorname{Im}\left(Z_{\text {in }}\right)\right)^{2}}}{\sqrt{\left(\operatorname{Re}\left(Z_{\text {in }}\right)+Z_{0}\right)^{2}+\left(\operatorname{Im}\left(Z_{\text {in }}\right)\right)^{2}}} \\
V S W R=\frac{1+|\Gamma|}{1-|\Gamma|}
\end{gathered}
$$

where $\mathrm{Z}_{0}$ is the characteristic impedance of the input coaxial line. $\operatorname{Re}\left(Z_{\text {in }}\right)$ and $\operatorname{Im}\left(Z_{\text {in }}\right)$ are the real component and the imaginary component of the input impedance, respectively.

\section{NUMERICAL ANALYSIS AND RESULTS}

In order to analyze the adapter proposed in this paper, the physical dimensions of the rectangular waveguide are fixed on the specific waveguide WR90, but the lengths and radius of the coupling loop are controlled in order to achieve a resistance of 50 ohms through the computer simulation of (1). Therefore, the input impedance of the end-launched adapter has to be matched to the characteristic impedance of the coaxial line filled with a material with a dielectric constant of 2.1 . 
To find the behavior of the input impedance, a commercial WR90 waveguide is selected and the lengths and radius are chosen to be in the ranges of $\mathrm{L}_{1}=11.4-15.4$ $\mathrm{mm}, \mathrm{L}_{2}=4.0-4.4 \mathrm{~mm}, \mathrm{c}_{\mathrm{i}}=0.5-1.5 \mathrm{~mm}$, and $\mathrm{x}_{0}=5.7-6.3 \mathrm{~mm}$, respectively. According to procedure of Fig. 2 and Table 1, the number of modes is extended up to the 9th higher order mode to ensure the convergence rate of the input impedance.

From the perspective of the above physical parameters of the adapter, the input impedance of the end-launched adapter can be matched from the above physical parameters of the adapter by properly choosing the length of the loop $\left(L_{1}, L_{2}\right)$, the radius of $c_{i}$, and the input feeding point of $x_{0}$. It is the ideal case that the resistive component of input impedance equals the characteristic impedance of the coaxial line and the reactive component equals zero to ensure the optimum design of the adapter. However, it is difficult in practice to ensure that the reactive component is zero over the whole waveguide band. Therefore, we have to have a trade-off in which the resistive component approaches the characteristic impedance of the coaxial line and also the reactive component approaches zero over the operating frequency band. This is done by controlling the physical parameters $\left(L_{1}, L_{2}, c_{i}, x_{0}\right)$ of the coupling loop of the end-launched adapter.

Figs. 3 and 4 show the evaluation of the input impedance and the input VSWR, respectively. The results of the proposed method, previous work, the HFSS, and the measured data have been compared with each other. The physical dimensions of the end-launched adapter have been determined to be $\mathrm{L}_{1}=13.4 \mathrm{~mm}, \mathrm{~L}_{2}=4.2 \mathrm{~mm}, \mathrm{c}_{\mathrm{i}}=1 \mathrm{~mm}$ and $\mathrm{x}_{\mathrm{o}}=5.9 \mathrm{~mm}$ under the best-matched condition below the VSWR $=3$. The comparison shows that the proposed result based on this letter for the input VSWR of the end-launched adapter agree well with the measured data and the $\operatorname{HFSS}(\Delta s=0.01)$ data over the wideband frequency from $7.5 \mathrm{GHz}$ to $10.6 \mathrm{GHz}$. However, in the results of [2, 4], the minimum VSWR are shown at $9.825 \mathrm{GHz}$ and $9.3 \mathrm{GHz}$ with bandwidths of $350 \mathrm{MHz}$ and $1.05 \mathrm{GHz}$, respectively. Alternatively, the results of the previous studies have a narrow bandwidth and also have discrepancies with the others.

Fig. 5 shows the photograph of the end-fed waveguide adapter combined with the WR90 waveguide with a coupling loop. This adapter is implemented by the copper metal and coaxial feeder line with the physical parameter dimensions of Fig. 4.

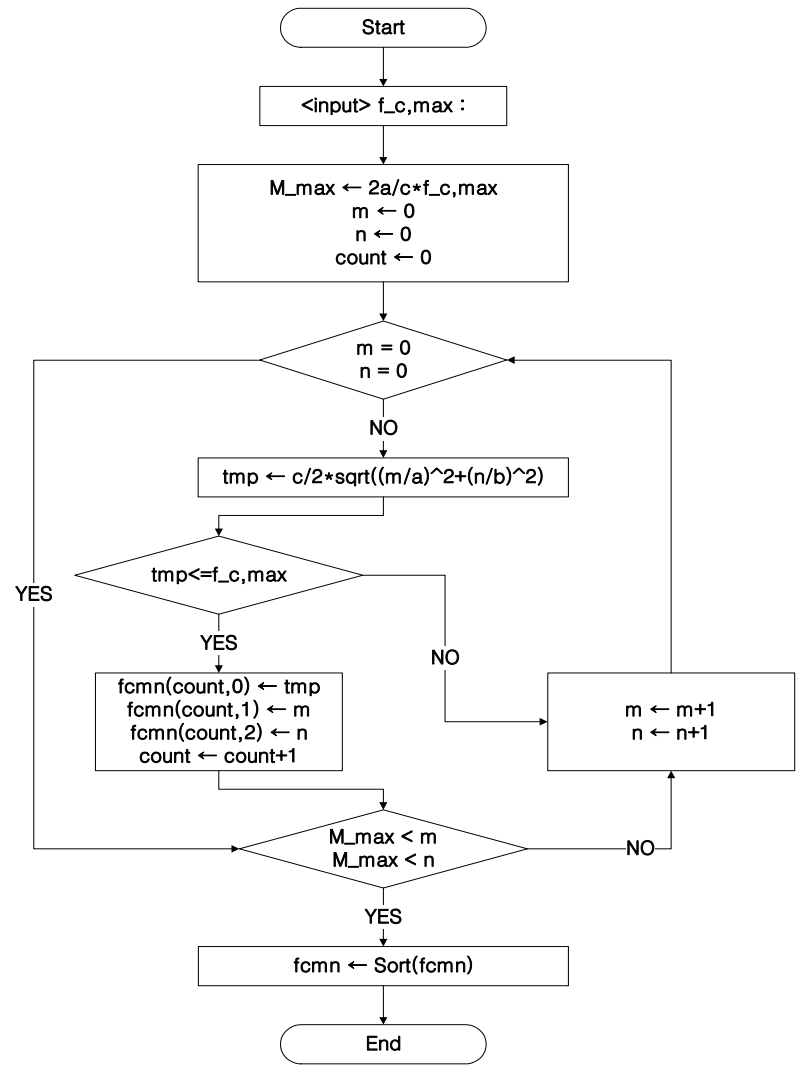

Fig. 2. Sorting flow chart of cut-off frequency (fcmn) and propagation mode number $(m, n)$ in waveguide.

Table 1. Higher order mode and cut-off frequency in waveguide

\begin{tabular}{ccc}
\hline $\begin{array}{c}\text { Waveguide mode } \\
(\mathrm{m}, \mathrm{n})\end{array}$ & Higher order mode (p) & Cut-off frequency (GHz) \\
\hline $\mathrm{TE}_{10}$ & $\mathrm{~m}=1, \mathrm{n}=0 \rightarrow \mathrm{p}=1$ & 6.557 \\
$\mathrm{TE}_{20}$ & $\mathrm{~m}=2, \mathrm{n}=0 \rightarrow \mathrm{p}=2$ & 13.114 \\
$\mathrm{TE}_{11}$ & $\mathrm{~m}=1, \mathrm{n}=1 \rightarrow \mathrm{p}=3$ & 16.145 \\
$\mathrm{TM}_{11}$ & $\mathrm{~m}=1, \mathrm{n}=1 \rightarrow \mathrm{p}=4$ & 16.145 \\
$\mathrm{TE}_{30}$ & $\mathrm{~m}=3, \mathrm{n}=0 \rightarrow \mathrm{p}=5$ & 19.172 \\
$\mathrm{TE}_{21}$ & $\mathrm{~m}=2, \mathrm{n}=1 \rightarrow \mathrm{p}=6$ & 19.740 \\
$\mathrm{TM}_{21}$ & $\mathrm{~m}=2, \mathrm{n}=1 \rightarrow \mathrm{p}=7$ & 19.740 \\
$\mathrm{TE}_{31}$ & $\mathrm{~m}=3, \mathrm{n}=1 \rightarrow \mathrm{p}=8$ & 24.590 \\
$\mathrm{TM}_{31}$ & $\mathrm{~m}=3, \mathrm{n}=0 \rightarrow \mathrm{p}=9$ & 24.590 \\
\hline
\end{tabular}




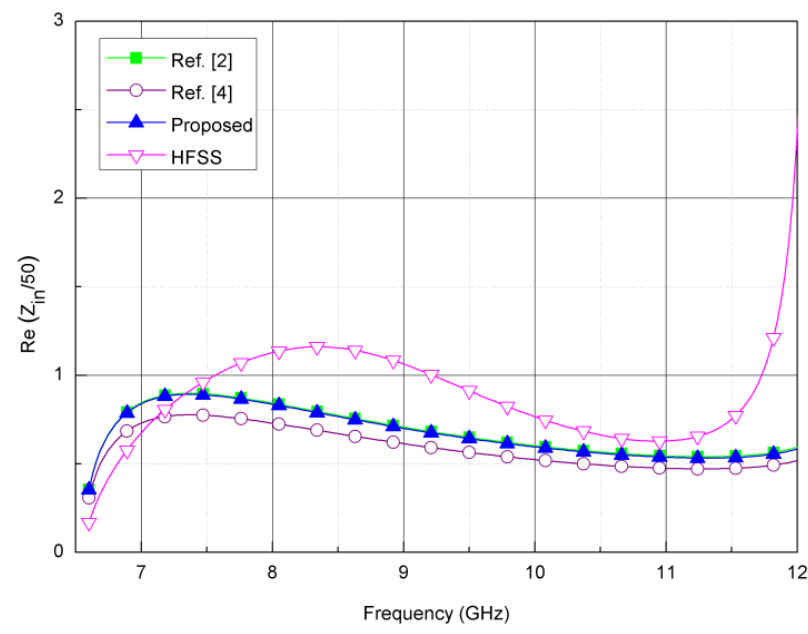

(a)

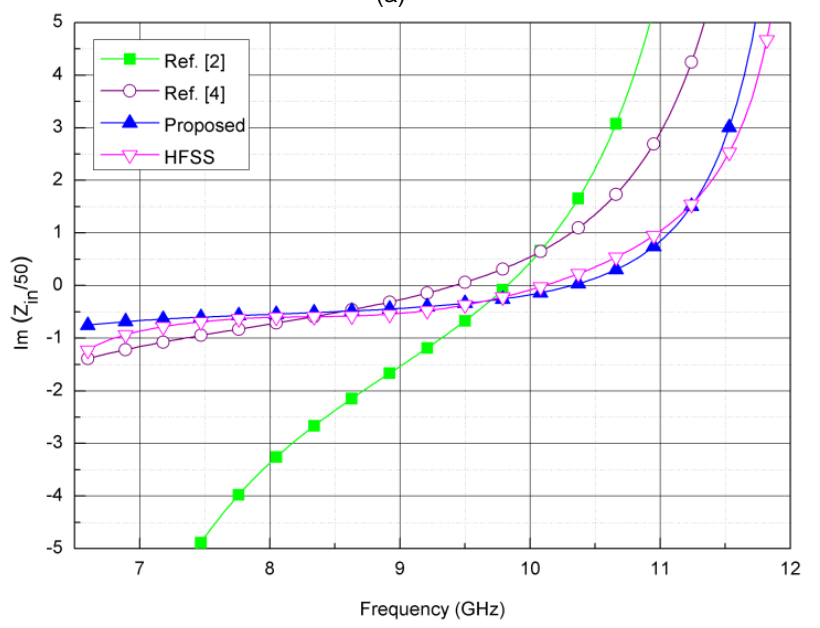

(b)

Fig. 3. Input impedance of end-launched adapter. (a) Resistive component (b) reactive component. HFSS: high frequency structure simulator.

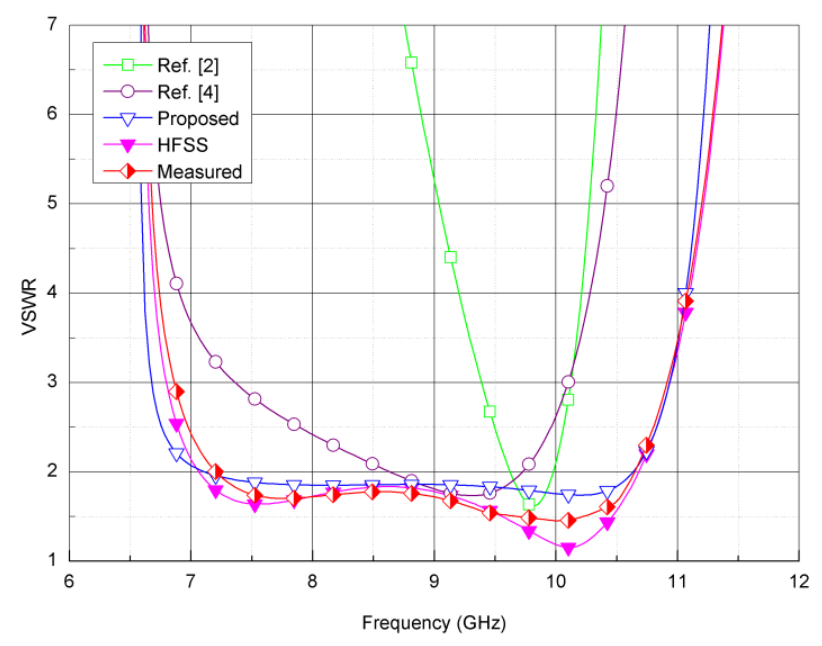

Fig. 4. Voltage standing wave ratio (VSWR) of end-launched adapter. HFSS: high frequency structure simulator.

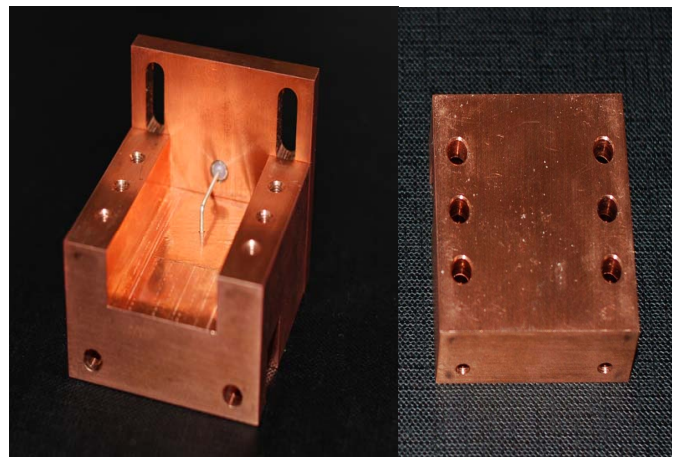

Fig. 5. Fabricated photograph of end-launched adapter.

\section{CONCLUSIONS}

The accurate expression of the input impedance for an analysis of the end-launched rectangular waveguide adapter has been presented. It is a more accurate formula that does not use an approximation scheme considering the input impedance as the dominant mode together with the higher order modes inside a waveguide adapter. The variation of the input impedance according to the design parameters of the coupling loop has also been described. The results obtained from numerical calculation through the use of proposed method were compared with previous work, and the validity of these results is verified by a commercial electromagnetic tool in HFSS. There is a good agreement between the proposed analytical results and the results in HFSS for the input VSWR in the wideband frequency range of $7.5 \mathrm{GHz}$ to $10.5 \mathrm{GHz}$. Therefore, the novel theoretical formula has more accuracy than the formulas derived in previous studies and allows the design to be based on a dependable quantitative criterion.

\section{REFERENCES}

[1] J. C. Dix, "Design of waveguide/coaxial transition for the band 2.5$4.1 \mathrm{Gc} / \mathrm{s}$," Proceedings of the Institution of Electrical Engineers, vol. 110, no. 2, pp. 253-255, 1963.

[2] M. D. Deshpande, B. N. Das, and G. S. Sanyal, “Analysis of an end launcher for an X-band rectangular waveguide,” IEEE Transactions on Microwave Theory and Techniques, vol. 27, no. 8, pp. 731-735, 1979.

[3] M. D. Deshpande and B. N. Das, "Analysis of an end launcher for a circular cylindrical waveguide," IEEE Transactions on Microwave Theory and Techniques, vol. 26, no. 9, pp. 672-675, 1978.

[4] S. M. Saad, "A more accurate analysis and design of coaxial-torectangular waveguide end launcher," IEEE Transactions on Microwave Theory and Techniques, vol. 38, no. 2, pp. 129-134, 1990. 
[5] R. Levy and L. W. Hendrick, "Analysis and synthesis of in-line coaxial-to-waveguide adapters,” IEEE MTT-S International Microwave Symposium Digest, Seattle, WA, pp. 809-811, 2002.

[6] D. H. Kim, J. W. Jwa, and D. Y. Yang, "An accurate analysis of a collinear end launcher waveguide adapter excited by loop coupling," 24th Annual Review of Progress in Applied Computational Electromagnetics, vol. 24, pp. 664-669, 2008.

[7] E. C. Jardan and K. G. Balmain, Electromagnetic Waves and Radiating Systems, Englewood Cliffs, NJ: Prentice-Hall, 1968.

[8] R. F. Harrington, Time-Harmonic Electromagnetic Fields, New York, NY: McGraw-Hill, 1961.

\section{APPENDIX}

$$
\begin{aligned}
A_{m n}= & \frac{1}{k^{2}-\left(\frac{n \pi}{b}\right)^{2}}\left[\frac{n \pi}{b k} \sin \left(\frac{n \pi L_{2}}{b}\right) \cos \left(k L_{2}\right)-\sin \left(k L_{2}\right) \cos \left(\frac{n \pi L_{2}}{b}\right)\right] \\
B_{m n, 1}= & e^{\gamma L_{1}}\left[2 \pi I_{0}\left(\gamma c_{i}\right) J_{0}\left(\frac{m \pi c_{i}}{a}\right)\right. \\
& \left.+4 \pi \sum_{s=1}^{\infty}\left\{(-1)^{s} \cos (s \pi) I_{2 s}\left(\gamma c_{i}\right) J_{2 s}\left(\frac{m \pi c_{i}}{a}\right)\right\}\right] \\
B_{m n, 2}= & e^{-\gamma L_{1}}\left[2 \pi I_{0}\left(-\gamma c_{i}\right) J_{0}\left(\frac{m \pi c_{i}}{a}\right)\right. \\
& \left.+4 \pi \sum_{s=1}^{\infty}\left\{(-1)^{s} \cos (s \pi) I_{2 s}\left(-\gamma c_{i}\right) J_{2 s}\left(\frac{m \pi c_{i}}{a}\right)\right\}\right] \\
C_{m n}= & j \omega \mu+\frac{1}{j \omega \varepsilon}\left(\frac{n \pi}{b}\right)^{2}
\end{aligned}
$$

$$
\begin{aligned}
D_{m n}= & \sin \left(\frac{m \pi x_{0}}{a}\right) e^{-\gamma L_{1}}\left[2 \pi I_{0}\left(-\gamma c_{i}\right) J_{0}\left(\frac{m \pi c_{i}}{a}\right)\right. \\
& \left.+4 \pi \sum_{s=1}^{\infty}\left\{(-1)^{s} \cos (s \pi) I_{2 s}\left(-\gamma c_{i}\right) J_{2 s}\left(\frac{m \pi c_{i}}{a}\right)\right\}\right] \\
E_{m n}= & \sin \left(\frac{m \pi x_{0}}{a}\right) \sin \left(\frac{n \pi L_{2}}{b}\right)\left[2 \pi J_{0}\left(\frac{m \pi x_{0}}{a}\right) J_{0}\left(\frac{n \pi L_{2}}{b}\right)\right. \\
& \left.+\sum_{s=1}^{\infty} \sum_{t=1}^{\infty}\left\{J_{2 s}\left(\frac{n \pi c_{i}}{b}\right)(-1)^{t} J_{2 t}\left(\frac{m \pi c_{i}}{a}\right) \frac{\sin (4(s-t) \pi)}{s-t}\right\}\right] \\
F_{m n, 1}= & \left(-j \omega \mu \gamma-\frac{\gamma k^{2}}{j \omega \varepsilon}\right) \frac{\sin \left(2 k\left(L_{1}+L_{2}\right)\right)-\sin \left(2 k L_{2}\right)+2 k L_{1}}{4 k} \\
F_{m n, 2}= & \left(j \omega \mu \gamma-\frac{\gamma k^{2}}{j \varpi \varepsilon}\right) e^{-\gamma L_{1}} \frac{\left(\gamma \cos \left(k L_{2}\right)+k \sin \left(k L_{2}\right)\right)}{2\left(k^{2}+\gamma^{2}\right)} \\
& {\left[\gamma e^{-\gamma L_{1}} \cos \left(k L_{2}\right)-\gamma \sin \left(k L_{2}\right)-2 k \sin \left(k\left(L_{1}+L_{2}\right)\right)\right.} \\
& \left.+k e^{\gamma L_{1}} \sin \left(k L_{2}\right)+k e^{-\gamma L_{1}} \sin \left(k L_{2}\right)\right] \\
F_{m n, 3}= & \frac{k \sin \left(k\left(L_{1}+L_{2}\right)\right)}{k^{2}+\gamma^{2}}\left(-j \omega \mu+\frac{\gamma^{2}}{j \omega \varepsilon}\right) \\
& {\left[k \sin \left(k\left(L_{1}+L_{2}\right)\right)+\gamma \cos \left(k\left(L_{1}+L_{2}\right)\right)\right.} \\
& \left.-\gamma e^{-\gamma L_{1}} \cos \left(k L_{2}\right)-k e^{-\gamma L_{1}} \sin \left(k L_{2}\right)\right]
\end{aligned}
$$

where $J_{n}$ and $I_{n}$ are the $\mathrm{n}^{\text {th }}$-order Bessel function of the first kind and the $n^{\text {th }}$-order modified Bessel function of the first kind, respectively.

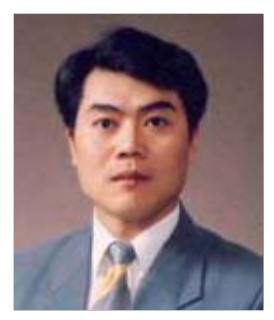

\section{Doo-Yeong Yang}

Dr. Yang received the B.S. in Telecommunication Engineering from Jeju National University, Korea in 1984, and also received the M.S. and Ph.D. in Electrical and Telecommunication Engineering from Hanyang University, Korea in 1989 and 1992, respectively. From January 2001 to August 2002, He was an international research professor, Utah State University in USA. Since 1992, he has been a professor in the Department of Telecommunication Engineering, Jeju National University, Korea. His current research interests include RF devices, microwave circuits, and wireless and satellite communication systems. 